\title{
Changes in functional connectivity in people with HIV switching antiretroviral therapy
}

\author{
Sofia Toniolo ${ }^{1,2} \cdot$ Mara Cercignani $^{1} \cdot$ Borja Mora-Peris $^{3,4}$ - Jonathan Underwood ${ }^{3}$. Jasmini Alagaratnam ${ }^{3}$. \\ Marco Bozzali $^{1}$ - Marta Boffito ${ }^{3,5} \cdot$ Mark Nelson $^{5} \cdot$ Alan Winston $^{3}$ • Jaime H. Vera ${ }^{6}$
}

Received: 13 January 2020 / Revised: 14 April 2020 / Accepted: 12 May 2020 / Published online: 4 June 2020

(C) The Author(s) 2020

\begin{abstract}
We assessed changes in functional connectivity by fMRI (functional magnetic resonance imaging) and cognitive measures in otherwise neurologically asymptomatic people with HIV (PWH) switching combination antiretroviral therapy (cART). In a prospective study (baseline and follow-up after at least 4 months), virologically suppressed PWH switched non-nuclease reverse-transcriptase inhibitors (NNRTI; tenofovir-DF/emtricitabine with efavirenz to rilpivirine) and integrase-strand-transfer inhibitors (INSTI; tenofovir-DF/emtricitabine with raltegravir to dolutegravir). PWH were assessed by resting-state fMRI and stop-signal reaction time (SSRT) task fMRI as well as with a cognitive battery (CogState ${ }^{\mathrm{TM}}$ ) at baseline and follow-up. Switching from efavirenz to rilpivirine $(n=10)$ was associated with increased functional connectivity in the dorsal attention network (DAN) and a reduction in SSRTs $(p=0.025)$ that positively correlated with the time previously on efavirenz (mean $=4.8$ years, $p=0.02$ ). Switching from raltegravir to dolutegravir $(n=12)$ was associated with increased connectivity in the left DAN and bilateral sensory-motor and associative visual networks. In the NNRTI study, significant improvements in the cognitive domains of executive function, working memory and speed of visual processing were observed, whereas no significant changes in cognitive function were observed in the INSTI study. Changes in fMRI are evident in PWH without perceived neuropsychiatric complaints switching cART. fMRI may be a useful tool in assisting to elucidate the underlying pathogenic mechanisms of cART-related neuropsychiatric effects.
\end{abstract}

Keywords HIV $\cdot$ fMRI $\cdot$ Neuropsychological assessment $\cdot$ Memory $\cdot$ Attention

Alan Winston and Jaime H. Vera contributed equally to this work.

Sofia Toniolo

sofia.toniolo@ndcn.ox.ac.uk

1 Clinical Imaging Sciences Centre, Brighton and Sussex Medical School, Knightsgate Road, Falmer Campus, Brighton BN1 9RR, UK

2 Present address: Nuffield Department of Clinical Neurosciences, University of Oxford, New Radcliffe House, Walton St., Oxford OX2 6BW, UK

3 Department of Infectious Disease, Faculty of Medicine, St Mary's Campus, Imperial College London, Praed Street, London W2 1NY, UK

4 Division of Infection and Immunity, School of Medicine, Cardiff University, UHW Main Building, Heath Park, Cardiff CF14 4XN, UK

5 Department of HIV Medicine, Chelsea and Westminster NHS Foundation Trust, 369 Fulham Road, London SW10 9NH, UK

6 Department of Global Health and Infection, Brighton and Sussex Medical School, Brighton BN1 9PX, UK

\section{Introduction}

Earlier treatment initiation and wider access to combination antiretroviral therapy (cART) have resulted in a significant decline in the incidence of HIV-associated central nervous system (CNS) diseases (Garvey et al. 2011). Despite this, neuropsychiatric events, such as cognitive deficits, anxiety, depression and insomnia, are frequently reported in people with HIV (PWH) on effective cART (Knights et al. 2017). Alongside traditional pathogenic mechanisms such as the legacy effect of HIV in CNS prior to cART initiation and CNS immune activation, antiretroviral toxicity may be a potential pathogenic factor responsible for the occurrence of these neuropsychiatric symptoms (Underwood et al. 2014). Indeed, efavirenz, a highly efficacious non-nucleoside reverse-transcriptase inhibitor (NNRTI), has been associated with neuropsychiatric symptoms in $20-40 \%$ of patients which may persist for several years after treatment initiation (Zhuang et al. 2017; Sacktor et al. 2006; McCutchan et al. 2007). Integrasestrand-transfer inhibitors (INSTI) have also been linked to an 
increased risk of neuropsychiatric adverse events, with dolutegravir being associated with the highest rate of discontinuation compared with other INSTI (2-6\%) (Elzi et al. 2017; Hoffmann et al. 2017). What impact antiretroviral drugs might have on brain activity and how it correlates with covert neuropsychiatric symptoms remains poorly described.

Functional MRI (fMRI) is an imaging modality that may provide valuable information on the effects of different cART regimens on the CNS by measuring changes in brain functional connectivity at rest or in response to tasks (Wise and Tracey 2006). The aim of this study is to assess changes in functional connectivity by fMRI and cognitive and behavioural measures in neurologically asymptomatic PWH participating in studies assessing changes in NNRTI and INSTI.

\section{Methods}

\section{Subject selection and study design}

PWH were enrolled in two separate phase IV, open-label studies conducted in 3 UK sites (Chelsea and Westminster Hospital, St. Mary's Hospital London and the Royal Sussex County Hospital) between January 2016 and January 2018.

Inclusion criteria for both studies included no history of subjective or objective neurological or psychiatric symptoms, age $\geq 18$ years, plasma HIV RNA $<40$ copies $/ \mathrm{mL}$ for at least 3 months, CD4 cell count $>50$ cells $/ \mu \mathrm{L}$ and being stable on the same cART regimen for at least 12 weeks (tenofovir-DF/ emtricitabine/efavirenz for the first study (NNRTI study) and tenofovir-DF/emtricitabine and raltegravir for the second study (INSTI study) (see Fig. 1).

Fig. 1 Study design for the NNRTI and INSTI studies

\section{a NNRTI study}

Subjects were excluded if they had previous exposure to rilpivirine in the NNRTI study and dolutegravir in the INSTI study, viral hepatitis co-infection, evidence of HIV drug resistance mutations, use of disallowed concomitant medication or current self-reported recreational drug use or alcohol abuse.

In the NNRTI study, all eligible participants $(n=10)$ were switched from the fixed-dose combination tenofovir-DF/ emtricitabine/efavirenz to tenofovir-DF/emtricitabine/ rilpivirine for 6 months. In the INSTI study, participants were randomized in a 1:2 fashion to either remain on the same cART drug regimen or switch to tenofovir-DF/emtricitabine and dolutegravir $50 \mathrm{mg}$ once daily for the duration of the study period (4 months). Randomization accounted for a total of 12 patients switching to dolutegravir and of 8 patients that remained on raltegravir. At each study visit, all study participants were specifically questioned and assessed by a research nurse or doctor about any psychiatric or neurological adverse events.

\section{Standard protocol approvals, registrations and patient consents}

The studies were conducted in accordance with the Declaration of Helsinki, the Uniform Requirements for Manuscripts submitted to Biomedical Journals, were approved by the research ethics committee, the UK regulatory authority and Medicines and Healthcare products Regulatory Agency (MRHA) and were registered with the EudraCT trials database (2014-002284-15) and (2014-003710-84). All subjects gave written informed consent prior to study initiation for both studies. The contracts between academic institutions and the funders stated clearly independence in terms of design,
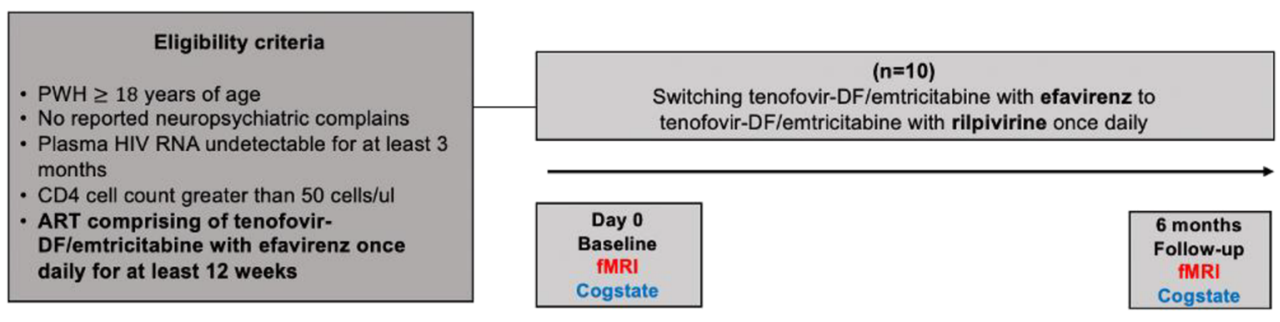

\section{b INSTI study}

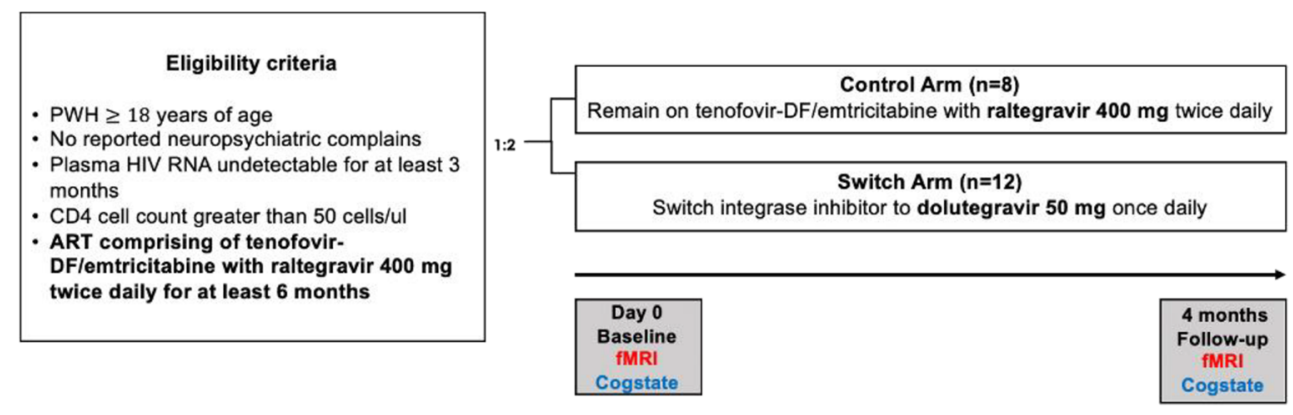


analysis and dissemination of the results. The present manuscript was analysed by investigators that have never received any funding from HIV-related pharmaceutical companies as described in the disclosures. Furthermore, the neuroimaging analysis was conducted blinded to the drug names to prevent the imaging specialist from bias.

\section{Study procedures}

\section{Clinical assessments}

Cognitive testing Cognitive testing was performed at baseline and at the final study visit using CogState ${ }^{\mathrm{TM}}\left(\mathrm{CogState}^{\mathrm{TM}} \mathrm{Ltd}\right.$., Melbourne, Australia), a computerized battery previously validated in PWH (Cysique et al. 2006) to assess processing speed, attention, visual learning, spatial problem solving, working memory and executive functions.

Cognitive results were compared with standardized normative data provided by $\operatorname{CogState}{ }^{\mathrm{TM}}$ and transformed into Zscores. Within the study time point, changes were tested using the Wilcoxon signed-rank test.

Patient-reported outcome measures Participants completed the following questionnaires at baseline and follow-up: Patient Health Questionnaire-9 (PHQ-9) (Kroenke et al. 2001), Beck's Depression Inventory (BDI) (Beck et al. 1961), Pittsburgh Sleep Quality Index (PSQI) (Buysse et al. 1989) and Hospital and Anxiety and Depression Scale (HADS) (Zigmond and Snaith 1983). Within the study time point, changes were tested using the Wilcoxon signed-rank test.

\section{Imaging assessments}

Resting-state network fMRI analysis Data pre-processing: All imaging was obtained using a 3.0T MR scanner (SIEMENS MAGNETOM Verio syngo MR B17) with a 32-channel head coil for both visits. The following scans were collected from each studied subject at baseline and follow-up: a highresolution 3D fluid-attenuated inversion recovery (FLAIR) sequence $(\mathrm{TE}=394 \mathrm{~ms}, \mathrm{TR}=5000 \mathrm{~ms}, \mathrm{TI}=1800 \mathrm{~ms}$, fieldof-view $=250 \mathrm{~mm}, 160$ contiguous slices of $1 \mathrm{~mm}$ thickness, voxel size $=1 \mathrm{~mm} 3$ ); a three-dimension (3D) magnetizationprepared rapid gradient-echo (MPRAGE) $(\mathrm{TE}=2.98 \mathrm{~ms}$, $\mathrm{TR}=2300 \mathrm{~ms}, \mathrm{TI}=900 \mathrm{~ms}$, flip angle $=9^{\circ}$, field-of-view $=$ $256 \mathrm{~mm}, 160$ contiguous slices of $1 \mathrm{~mm}$ thickness, voxel size $=1 \mathrm{~mm} 3$ ); and a BOLD response-sensitive echo-planar imaging (EPI) sequence $(\mathrm{TE}=30 \mathrm{~ms}, \mathrm{TR}=2000 \mathrm{~ms}$, flip angle $=80^{\circ}$, field-of-view $=192 \mathrm{~mm}, 35$ interleaved slices of $3 \mathrm{~mm}$ thickness with no gap, voxel size $=3 \mathrm{~mm} 3$, duration $10.06 \mathrm{~min}$ ) for resting-state fMRI. Participants were instructed to remain still with their eyes closed and not think of anything specific while trying to stay awake. Nine out of 10 in the
NNRTI study, 9 out of 11 people on the dolutegravir arm and 7 out of 8 on the raltegravir arm in the INSTI study gave consent to perform the fMRI task so an additional sequence with the same parameters as the resting state was acquired while participants performed the stop-signal task (SST) behavioural task. According to the inclusion criteria, FLAIR scans were reviewed to exclude the presence of significant macroscopic brain abnormalities. For all MRI scans, restingstate fMRI data analysis was carried out using FMRIB Software Library (FSL) (Jenkinson et al. 2012). We first discarded the first 4 volumes to allow steady-state magnetization. Each dataset underwent motion correction with MCFLIRT (Jenkinson et al. 2002a), non-brain tissue removal with brain extraction tool (BET) (Jenkinson et al. 2002b), nonlinear registration of the functional images to the main structural image with FNIRT (FMRIB's nonlinear image registration tool) and denoising using independent component analysis-based Automatic Removal Of Motion Artifacts (ICA-AROMA) (Pruim et al. 2015). The images extracted from AROMA were then normalized to the Montreal Neurological Institute (MNI) standard space ( $2 \mathrm{~mm}$ isotropic). Grand-mean intensity normalization and spatial smoothing with a Gaussian kernel of $5.0 \mathrm{~mm}$ full width at halfmaximum (FWHM) were subsequently performed.

fMRI RSN first-level analysis: A group-level ICA was performed across all subjects and all scans using MELODIC (Beckmann and Smith 2004). First, the previously preprocessed $4 \mathrm{D}$ dataset was temporally transformed by concatenation into a single time series. This new 4D image was then separated into different independent components (ICs).

We decided to use 2 different IC dimensionalities (number of IC components) for maximizing the matching between the data generated by our sample and previously described RSNs. In the NNRTI study, we did not a priori set the number of component, and within the 38 group-level IC maps originated by MELODIC, we selected 8 cognitively relevant networks, i.e. default mode network (DMN), executive network (EXE), dorsal attention network (DAN), ventral attention network (VAN), anterior limbic network (ANTLIMB), posterior limbic network (POSTLIMB) and left and right frontoparietal network (LFP and RFP).

For the INSTI study, we set the ICs to 20 according to the 20 RSN model by Smith et al. (Smith et al. 2009), and within the group-level IC maps originated by MELODIC, we selected 10 networks of interest, i.e. DMN, EXE, salience network (SAL), DAN, VAN, limbic network (LIMB), LFP and RFP, sensory-motor network (SM) and associative visual network (VISAS). The identification of each network was defined according to previously described RSNs (Smith et al. 2009; Thomas Yeo et al. 2011; Dipasquale and Cercignani 2016; Laird et al. 2011), with the associative visual network corresponding to the lateral visual areas by Smith et al. (Smith et al. 2009). A visual inspection of each component spatial profile 
to ensure the effectiveness of the matching between the RSNs originated by MELODIC and previously described RSNs was performed. This procedure allows us to check which RSN model describes best the networks generated by our data sample.

fMRI RSN second-level analysis: Subject-specific IC maps were obtained using dual regression (Filippini et al. 2009) and, subsequently, a second-level analysis testing the betweensession group difference was performed using FSL randomize (Winkler et al. 2014) through a 2-sample $t$ test on the betweensession difference image with the number of iterations set to 2000. A binary mask of every network was obtained by a onesample $t$ test and the analysis was restricted within the mask. We estimated 4 contrasts: within-session means and the within-subject, between-session differences. The second-level inference was thresholded at a family-wise error (FWE)corrected probability of $p<0.05$ with threshold-free cluster enhancement (TFCE).

Stop-signal task $\mathrm{fMRI}$ analysis Subjects performed a response inhibition task, the stop-signal task (SST) inside the scanner as task fMRI at baseline and follow-up (see Fig. 2). The SST measures the efficiency of response inhibition, with lower SSRTs (stop-signal response times) indicating less time required to inhibit a response (Rae et al. 2014).

Briefly, participants performed a speeded response task (e.g. indicating whether an arrow points to the left or right). On "GO" trials, an arrow pointing to the left or right was presented, and the subjects were instructed to respond as

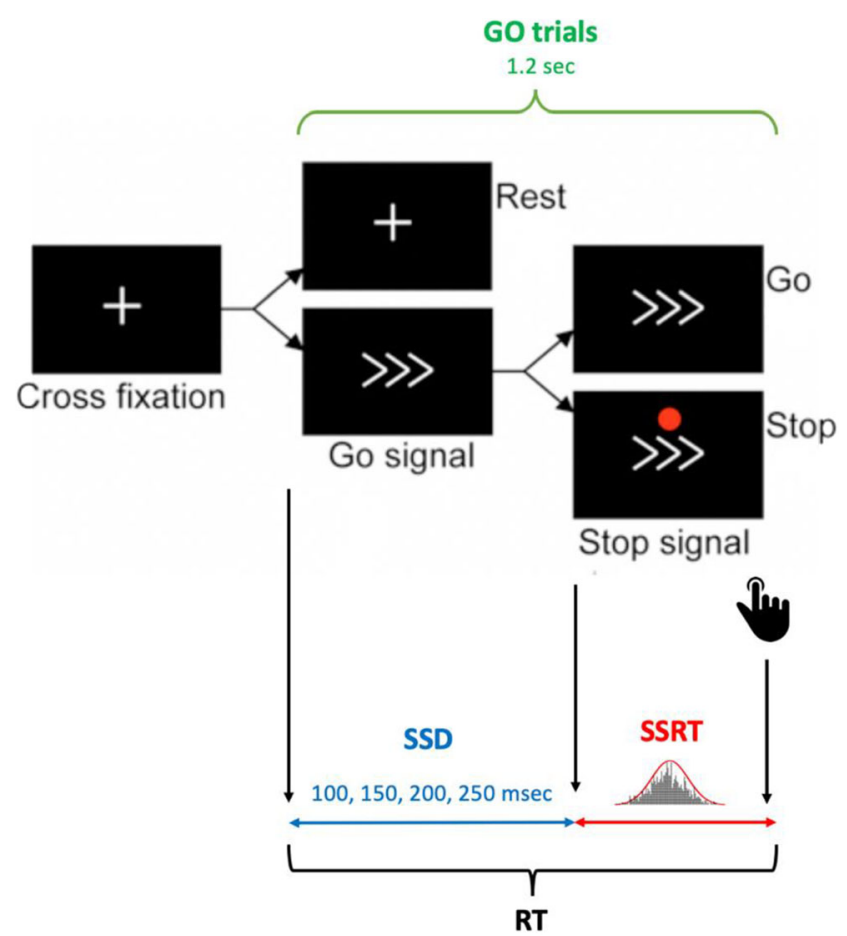

Fig. 2 Behavioural task (SST) experimental model. SSD, stop-signal delay; RT, response time; SSRT, stop-signal response time quickly as possible. On "STOP" trials, a stop signal was presented concurrently with or just after the target, and participants had to withhold their response. The onset time of the stop signal relative to the go stimulus or stop-signal delay (SSD) changed dynamically throughout the experiment and the efficiency of response inhibition was estimated by observing the effects of varying the SSD on the SSRT.

SSD changed dynamically throughout the experiment depending on the subject's performance, increasing by $50 \mathrm{~ms}$ if the subject inhibited successfully on a STOP trial or conversely decreasing the SSD by $50 \mathrm{~ms}$ if inhibition was unsuccessful. Four step-up and step-down algorithms (staircases), with initial SSD values of 100, 150, 200 and $250 \mathrm{~ms}$ respectively, were used to ensure convergence to inhibition of $50 \%$ trials by the end of the experiment. Average SSD was computed, for each subject, using the values of the four staircases after the subject had converged on 50\% inhibition (Aron and Poldrack 2006), while SSRT was estimated by subtracting average SSD from median correct RT in the GO trials. To improve subject attentive engagement and motivation over time, a "negative feedback" cue was presented based on whether the last reaction time (RT) was over $0.95 \%$ of the previous RTs. SSRT, RT and accuracy were compared between time points. Firstly, we used the Shapiro-Wilk test to assess normality distribution and then either a paired $t$ test or a Mann-Whitney $U$ test according to the normality of the data.

We then looked at associations between SSRT, RT and accuracy and clinical parameters. As most of the participants in both studies were of the male gender, no associations between gender and SSRT/RT were investigated. No significant associations between SSRT/RT parameters and age were observed. We selected 5 covariates of interest (years with HIV, years on cART, CD4 count, CD4/8 ratio and years on the drug) and we performed an ANOVA with repeated measures on SSRTs, RTs and accuracy using these specific clinical data as covariates.

Data pre-processing and fMRI SST first-level analysis: All image processing was done in Statistical Parametric Mapping Version 12 (SPM12). The first 4 images were discarded in order to allow steady-state magnetization. Images were realigned to the mean image, slice time corrected, normalized to the MNI standard template and smoothed with a Gaussian kernel of $5.0 \mathrm{~mm}$ FWHM.

The following contrasts were estimated and taken to the second level for each session: STOP correct trials $>$ GO trials and GO trials > STOP correct trials.

fMRI SST second-level analysis: For the NNRTI study, we performed a paired $t$ test between images at baseline and after 6 months. For the INSTI study, the between-session difference image was assessed using ImCalc SPM function in order to obtain the final within-subjects' image and a two-sample $t$ test was used to assess the between-group difference in the 2 groups. 
For all results, we examined two different contrasts, respectively assessing whether participants showed increased or decreased fMRI activation between sessions (NNRTI study) or between groups (INSTI study) for both conditions: STOP > $\mathrm{GO}$ trials and $\mathrm{GO}>$ STOP trials. The resulting statistic images were thresholded at $p<0.05$ cluster-based FWE with cluster formed using $p<0.001$ at the voxel level.

Data availability statement The full anonymized dataset for this project will be deposited in the University of Sussex research repository as part of the University's Research Data Management Policy where all data for this study can be accessed upon request and approval by the research ethics committee.

\section{Results}

Ten participants from the NNRTI study and 20 participants from the INSTI study ( 8 in the control arm and 12 in the switch arm) completed study procedures. Baseline characteristics are presented in Table 1.

One MRI scan in the switch arm of the INSTI study at follow-up was corrupted by severe artefacts, so the subject was excluded from the group MRI analysis. Study drugs were well tolerated with all patients reporting over $95 \%$ adherence to therapy. No safety or laboratory concerns related to any of the study drugs were observed. At 4 months, plasma HIV RNA was $<40$ copies/mL in all subjects for both studies.

\section{Effect of switching cART on cognitive testing}

In the NNRTI study, no statistically significant differences between the time points were found in global cognitive scores; however, a significant improvement in cognitive tests assessing executive functions, working memory and visual-motor control were observed (Groton Maze Learning Test, One Back Test and Groton Maze Chase Test) (see Table 2). No changes in individual cognitive tests were observed in the INSTI study.

\section{Effect of switching CART on PROMs}

In the NNRTI study, a 3\% improvement in sleep quality (median change (IQR) from baseline: 3 ( -2 to 10$) ; p=0.009$ for the PSQI) and a $4.8 \%$ improvement in anxiety symptoms (median change (IQR) from baseline: 4.8 ( -4.8 to 33$)$; $p=$ 0.053 for HADS) at follow-up were observed following the switch. No differences in changes of other PROMs were observed and no statistically significant differences were observed in the INSTI study.

\section{Effect of switching CART on resting-state networks fMRI}

In the NNRTI study, over the study period, we observed significantly enhanced connectivity of the dorsal attention network (DAN), and in particular of the right superior parietal lobule (see Fig. 3). No other changes in functional connectivity among the other 7 networks examined were observed.

In the INSTI study, significantly increased connectivity in the DAN, VISAS (associative visual) and SM (sensory-motor) networks was observed over the study period (see Fig. 4). Within the DAN, the brain areas that showed an increase in functional connectivity were the left middle frontal gyrus (within the frontal eye fields), left superior parietal lobule, left supramarginal gyrus and left superior frontal gyrus. Regarding the VISAS, we found an enhanced connectivity within the right lateral occipital cortex. In the SM network, we found a wider increase of functional connectivity bilaterally in the superior and medial frontal gyrus and precentral gyrus.

\section{Effect of switching CART on behavioural measures (SST)}

In the NNRTI study, a reduction in SSRT was observed after switching from efavirenz to rilpivirine $(p=0.025)$. Participants showed good response accuracy in both conditions, thus confirming good compliance to the task. The

Table 1 Demographic characteristics for NNRTI and INSTI studies

\begin{tabular}{|c|c|c|c|}
\hline \multirow[t]{2}{*}{ Parameter } & \multirow[t]{2}{*}{ NNRTI study (efavirenz to rilpivirine) } & \multicolumn{2}{|c|}{ INSTI study (raltegravir to dolutegravir) } \\
\hline & & Control arm & Switch arm \\
\hline Number & 10 & 8 & 12 \\
\hline Age, years median (IQR) & $52(14)$ & $39.5(15.5)$ & $43(13)$ \\
\hline Male, $n(\%)$ & $10(100)$ & $7(87.5)$ & $12(100)$ \\
\hline White ethnicity, $n(\%)$ & $10(100)$ & $5(62.5)$ & $9(69.2)$ \\
\hline CD4+ count at study entry (cells/ $\mu \mathrm{L})$, median $(\mathrm{IQR})$ & $600(256)$ & $688(395)$ & $736(237)$ \\
\hline HIV RNA viral load $<40$ copies/mL, $n(\%)$ & $10(100)$ & $8(100)$ & $12(100)$ \\
\hline
\end{tabular}

Demographic characteristics for NNRTI and INSTI studies 
Table 2 Changes in cognitive function by study arm (NNRTI study)

\begin{tabular}{|c|c|c|c|c|c|c|}
\hline \multirow[b]{2}{*}{ Cognitive test } & \multirow[b]{2}{*}{ Cognitive domain } & \multirow[b]{2}{*}{ Outcome measure } & \multirow{2}{*}{$\begin{array}{l}\text { Baseline } \\
Z \text {-scores } \\
\text { (SD) }\end{array}$} & \multirow{2}{*}{$\begin{array}{l}6 \text { months } \\
Z \text {-scores } \\
\text { (SD) }\end{array}$} & \multirow{2}{*}{$\begin{array}{l}\text { Changes at } 6 \text { months from } \\
\text { baseline } \\
\text { Mean score difference }(95 \% \mathrm{CI})\end{array}$} & \multirow[t]{2}{*}{$\begin{array}{l}p \\
\text { value }\end{array}$} \\
\hline & & & & & & \\
\hline Identification & Attention & $\begin{array}{l}\text { Speed of performance } \\
\log 10 \text { milliseconds }\end{array}$ & $-0.39(0.48)$ & $-0.34(0.81)$ & $0.05(-0.80 / 0.91)$ & 0.88 \\
\hline Detection & $\begin{array}{l}\text { Psychomotor } \\
\text { function }\end{array}$ & $\begin{array}{l}\text { Speed of performance } \\
\log 10 \text { milliseconds }\end{array}$ & $-0.65(0.93)$ & $-0.72(0.64)$ & $-0.07(-0.5 / 0.42)$ & 0.75 \\
\hline One Card Learning & Visual learning & $\begin{array}{l}\text { Accuracy of } \\
\text { performance }\end{array}$ & $-0.66(1.09)$ & $-0.62(1.6)$ & $0.03(-1.11 / 1.18)$ & 0.94 \\
\hline One Back & Working memory & $\begin{array}{l}\text { Speed of performance } \\
\log 10 \text { milliseconds }\end{array}$ & $-0.01(0.97)$ & $-2.13(1.02)$ & $-2.11(-2.7 /-1.4)$ & 0.001 \\
\hline Groton Maze Chase & Visual-motor control & Moves per second & $0.10(1.02)$ & $-0.61(0.9)$ & $-0.72(-1.20 /-0.20)$ & 0.011 \\
\hline Groton Maze Learning & Executive function & Total number of errors & $-0.06(0.98)$ & $-2.13(1.2)$ & $-2.06(-2.90 /-1.22)$ & 0.003 \\
\hline SET shifting & Executive function & Total number of errors & $-0.04(0.96)$ & $0.03(1.02)$ & $0.07(-0.64 / 0.80)$ & 0.81 \\
\hline $\begin{array}{l}\text { Global cognitive } \\
Z \text {-score }\end{array}$ & Global cognition & $Z$-score & $-0.19(24)$ & $-0.58(0.78)$ & $-0.24(-1.05 / 0.5)$ & 0.24 \\
\hline
\end{tabular}

$S D$, standard deviation; $C I$, confidence interval; $p$ value, two-tailed. Lower scores at Identification, Detection, One Back, Groton Maze Chase, Groton Maze Learning, SET shifting, Global cognitive score indicate better performance. Higher scores at the One Card Learning task indicate better performance

reduction in SSRTs between the two conditions was positively correlated with the duration of efavirenz exposure, $F(1,3)=$ $20.976, p=0.020, \eta^{2}=0.875$. No statistical correlation between the other values and changes in SSRTs was observed (see Table 3). In the INSTI study, SSRTs $(p=0.606)$, mean RTs ( $p=0.724)$ and accuracy ( $p=0.252)$ were not significantly different between the two groups or between time points.

\section{Effect of switching CART on response inhibition in fMRI SST}

In both studies, the main effects of activation patterns for both contrasts were in line with previous meta-analysis data on the SST task. In the NNRTI study, a reduction in BOLD activation in the STOP > GO contrast was observed after switching efavirenz to rilpivirine in the right IFG (pars opercularis), right insula, right prefrontal cortex, right posterior parietal cortex, right lateral occipital gyrus and left primary, and associative auditory cortex and left primary motor cortex, but these results did not survive after correction for multiple comparisons (FWE). This was also the case for the inverse contrast rilpivirine to efavirenz, where BOLD activation was observed in the right paracingulate cortex, right IFG (pars orbitalis and pars opercularis), bilateral frontal eye fields, right middle frontal gyrus, right pre-SMA, left posterior parietal cortex, right lateral occipital gyrus and right angular gyrus. The reverse contrast, GO > STOP, did not show any significant result. In the INSTI study, the analysis did not show any area of increased or decreased connectivity between time points in $\mathrm{GO}>\mathrm{STOP}$ or STOP $>$ GO contrasts.

\section{Discussion}

Using a combined fMRI and cognitive behavioural approach, we were able to identify a dysfunction of the attentional network and executive functions that were restored by drug switching. From a neuropsychological perspective, in the NNRTI study, despite participants being asymptomatic, a significant improvement in sleep quality (measured by the PSQI) and anxiety symptoms (HADS) was observed, while in the INSTI study, we found no changes in any of the PROMs, which is in line with previous data (Mora-Peris et al. 2019), suggesting the observed changes are not just an artefact of repeated measurement. Moreover, these results

Fig. 3 Resting-state connectivity after switching to rilpivirine compared with the baseline. Red: areas of enhanced connectivity within the DAN (right superior parietal lobule). Blue: DAN mask. L, left; R, right

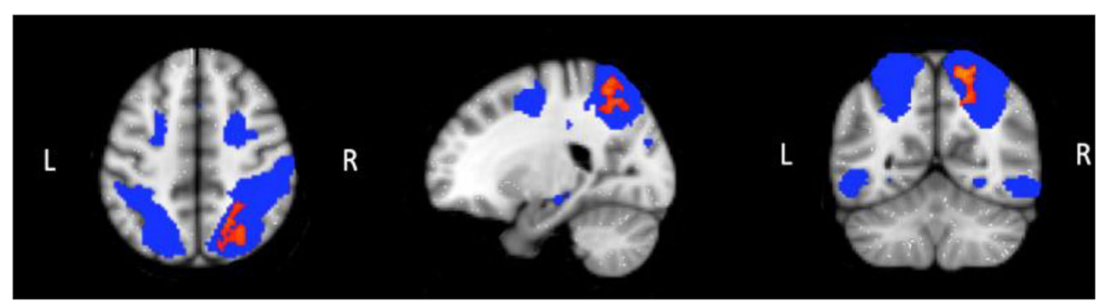


Fig. 4 Resting-state connectivity after switching to dolutegravir compared with the baseline. a DAN: red, areas of enhanced connectivity within the DAN (left middle frontal gyrus, left superior parietal lobule, left supramarginal gyrus and the left superior frontal gyrus). Orange, DAN mask. b VISAS: green, areas of enhanced connectivity within the VISAS network (right lateral occipital cortex). Magenta, VISAS mask. c SM: blue, areas within the SM network (superior and medial frontal gyrus and precentral gyrus) of enhanced connectivity. Pink, SM mask. L, left; R, right

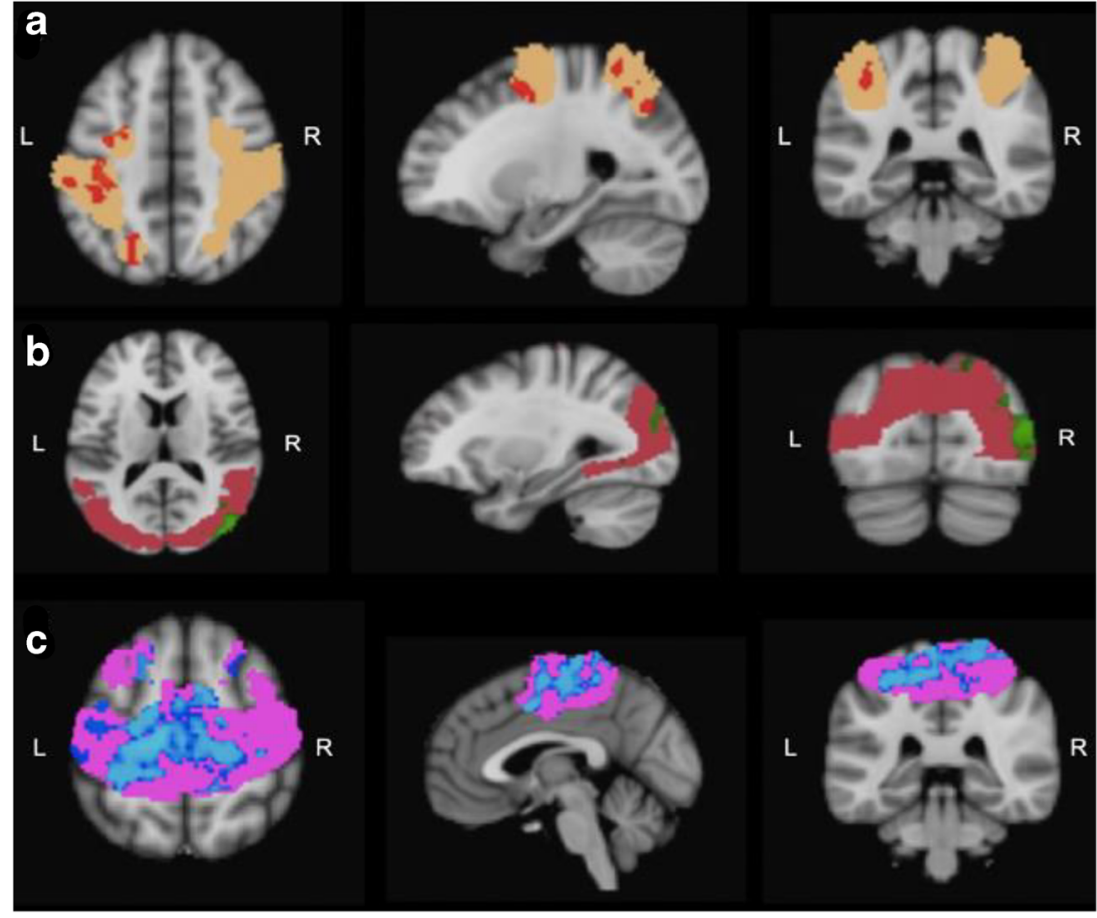

are in line with previous data reporting that switching from efavirenz containing to efavirenz-free regimens has a beneficial impact on sleep quality and anxiety, as well as on executive function and attention (Vera et al. 2019; Walker and Brown 2018; Knoch et al. 2006). Interestingly, executive function was also found to be positively affected by drug switching in our study, measured by an improvement on the Groton Maze Learning Test and moreover on the SST task, which has become a standard measure of response inhibition (Logan and Cowan 1984). Data on SST performance in PWH are scarce, though a recent study reported that drug-naïve PWH showed comparable SSRTs with respect to healthy controls but longer RTs (Du Plessis et al. 2015). In the NNRTI study, we found that SSRTs were longer in participants on efavirenz before switching to rilpivirine, suggesting a detrimental effect caused by efavirenz with a loss of correct response inhibition, which might be restored by switching to rilpivirine. Moreover, we observed that the change in SSRTs between the two conditions was directly correlated with the duration of efavirenz treatment. The longer the patients were on efavirenz, the longer the SSRTs were and the worst the efficacy of response inhibition was. We postulate that this might reflect a cumulative effect of efavirenz treatment on the ability to correctly inhibit responses.

Functional connectivity has been extensively used in HIV clinical research settings (Ernst et al. 2002; Chang et al. 2001; Schweinsburg et al. 2012). Nevertheless, studies on druginduced changes on resting-state networks in HIV populations are limited. If cART as a whole is taken into consideration, drugnaïve PWH show a lower functional connectivity in the DMN compared with healthy controls that could be restored after 12 weeks of cART (Zhuang et al. 2017). Interestingly, other experimental techniques such as MEG report that even PWH on effective cART show a disruption of the DMN compared with healthy controls (Becker et al. 2013), which could be explained by either the beneficial effect of cART being limited in restoring the network dysfunction produced by HIV infection or cART exerting both positive and negative effects on this RSN. Besides the DMN, increasing observations point towards an
Table 3 Association between clinical data and behavioural results for the NNRTI study

\begin{tabular}{lllll}
\hline & Mean values (SD) & SSRT $(p)$ & RT $(p)$ & Accuracy $(p)$ \\
\hline Years with HIV & $14.0(5.2)$ & 0.99 & 0.645 & 0.59 \\
Years on efavirenz & $4.8(3.1)$ & 0.02 & 0.18 & 0.20 \\
CD4 count (cells $/ \mu \mathrm{L})$ & $601.7(168.7)$ & 0.38 & 0.35 & 0.56 \\
CD8 count (cells $/ \mu \mathrm{L})$ & $551.6(150.8)$ & 0.89 & 0.35 & 0.56 \\
CD4/CD8 ratio & $1.1(0.3)$ & 0.51 & 0.35 & 0.69 \\
\hline
\end{tabular}

Years with HIV and years on efavirenz are expressed in years, CD4 and CD8 count in (cells $/ \mathrm{L}) .(p), p$ value, obtained using a repeated-measure ANOVA 
important role of the DAN in HIV-associated cognitive disorders (Ernst et al. 2002). The DAN is responsible for covert spatial attention, saccade planning and visual working memory (Vossel et al. 2014). Several fMRI studies have shown a greater loaddependent increase in activation in brain regions related to visual attention tasks (especially in the prefrontal and posterior parietal cortex) in PWH compared with healthy controls (Chang et al. 2008; Ernst et al. 2009), suggesting an increased brain metabolism due to an enhanced neuronal activity that could compensate for the underlying injury to the neural substrate caused by HIV infection. The driver of this attentional overload in PWH is largely unknown, and our study is one of the first to specifically assess the issue of the changes exerted by different cART regimens on the DAN.

In the NNRTI study, in PWH switching to an efavirenz-free cART, we observed improved attention through changes in the DAN. Specifically, our data indicate that the right superior parietal lobule (SPL) is the brain area which shows the highest vulnerability to efavirenz-induced damage. The role of the SPL in visuospatial attention has been previously reported by independent groups and different techniques (Fink et al. 2000; Fink et al. 2001; Wu et al. 2016; Vallar et al. 2014) and shows clear right lateralization, with the right posterior SPL showing stronger anatomical connections with the ipsilateral middle frontal gyrus (MFG) and inferior frontal gyrus (IFG), and with the contralateral posterior parietal cortex (PPC) than the left posterior SPL (Wu et al. 2016), accounting for the right predominance of visuospatial attention control. Interestingly, recent fMRI and MEG studies showed that the right SPL expresses the highest attentional load effect both in healthy controls and PWH under cART (Chang et al. 2008) and correlates with cognitive impairment in PWH (Becker et al. 2013). Therefore, the clear right lateralization of our finding is not surprising, though a bigger sample would be required to confirm its specificity. It is possible that efavirenz may cause a dysfunction of the DAN that is ameliorated, at least partially, by switching to rilpivirine. Moreover, since the DAN is activated during top-down feature-based attention, this would well reflect the improvements in the Groton Maze Learning Test, Chase Test and One Back Test. Therefore, while our results are in line with previous data on the role of the DAN dysfunction in PWH, we were able to dissect the differential impact that specific drugs can exert on this RSN.

The involvement of the VISAS, which is responsible for higher-level visual processing such as viewing complex stimuli and discrimination of locations in space, is not a surprising finding, given the fact that it constitutes one of the main projections to the DAN. Indeed, in the INSTI study, the finding of an enhanced connectivity in the left regions of the DAN and the right VISAS which are functionally highly correlated to the right regions of the DAN may suggest a compensatory mechanism due to an underlying damage of visuospatial processing and perceptual abilities. Sensory-motor function has been traditionally regarded as significantly impaired in PWH, especially in more severe cases and in the pre-cART era (Ances and Clifford 2008). However, it is still largely unknown the impact of different cART regimens on this network and further studies are needed in order to validate the relevance of this result.

There are limitations to our work. First, there is no HIVnegative control arm, and therefore, a comparison between subjects switching NNRTI and INSTI and healthy controls would have strengthened our interpretation of the comparisons found. However, the INSTI group findings, where no change was observed, would suggest that the NNRTI findings were the result of the switch form from efavirenz to rilpivirine rather than a repeated measures effect. Although De Plessis et al. (Du Plessis et al. 2015) showed comparable SSRTs between healthy controls and drug-naïve PWH, no study is currently available on SSRT differences between healthy controls and people PWH on efavirenz or rilpivirine containing cART. Secondly, the PROMs are self-reported questionnaires, so we cannot fully exclude a response shift bias, as is an intrinsic limitation of all self-reported questionnaires. Thirdly, these results should be considered exploratory, given the small sample size may limit our power of observation. As an example, we have applied fMRI power (Mumford 2012) to our current results in the NNRTI study to estimate the sample required to detect a difference in the insula for the contrast STOP $>$ GO. We estimated that for $80 \%$ power, a group of 30 participants should be sufficient. Moreover, it is known that physiological noise such as cardiac and respiratory signals can bias fMRI results. Data required for sophisticated correction procedure such as physiological noise model (PNM) was not available for this study, and we based our denoising procedures on ICAAROMA and visual assessment of noise components. Given that our areas of interest are far from the anatomical structures that are most susceptible to these artefacts (e.g. brainstem), we are confident that our data reflect a neural effect. Finally, we used 2 slightly different approaches for the ICAs of the 2 datasets: while in the INSTI study, we a priori set the number of components at 20; in the NNRTI, we let this number to be determined by the algorithm. Both approaches are valid and used within the neuroimaging community; in our case, the specific method was chosen to ensure the best fitting of our data to previously described RSNs. From a clinical perspective since attention disturbances, insomnia and anxiety are among the most common side effects of both efavirenz and dolutegravir and account for a high degree of therapy discontinuation (Apostolova et al. 2015; Hoffmann et al. 2017), we believe that the improvements in PSQI and HADS could play a major role in increasing medication adherence, while we argue that the change in functional connectivity of the DAN, VISAS and sensory-motor network could explain the reported lack of concentration and slower psychomotor function. 


\section{Conclusion}

In conclusion, evidence that fMRI changes are detectable in virologically suppressed, neurologically asymptomatic PWH on cART provides encouraging data on the potential use of these techniques as possible biomarker of CNS injury related to cART.

Acknowledgements The authors thank Rae Charlotte, PhD (Brighton and Sussex Medical School, Brighton, UK) for her support in SST fMRI conceptual modelling.

Authors' contributions T.S. performed the MRI analysis and analysed the behavioural data. W.A. and V.J.H. designed and conceptualized this study. C.M. and B.M. provided technical support and conceptual guidance for the MRI analysis. M.-P.B., A.J., B.M., U.J., W.A. and V.J.H. contributed to study idea and data collection. N.M. contributed to the study idea, wrote the grant and obtained funding for the NNRTI study. T.S., C.M., V.J.H. and W.A. drafted the manuscript. All authors revised the manuscript.

\section{Compliance with ethical standards}

The studies were conducted in accordance with the Declaration of Helsinki, the Uniform Requirements for Manuscripts submitted to Biomedical Journals, were approved by the research ethics committee, the UK regulatory authority and Medicines and Healthcare products Regulatory Agency (MRHA) and were registered with the EudraCT trials database (2014-002284-15) and (2014-003710-84).

Conflict of interest Sofia Toniolo, Mara Cercignani and Borja MoraPeris report no disclosures. Jonathan Underwood reports travel and honoraria from Gilead Sciences. Jasmini Alagaratnam reports grants to attend conferences from Gilead and MSD. Marco Bozzali reports honoraria and travel support from Lilli, Biogen and Roche. Marta Boffito reports travel and research grants from and has been speaker/advisor for Janssen, Roche, ViiV, Bristol-Myers Squibb, Merck Sharp \& Dohme, Gilead, Mylan, Cipla and Teva. Mark Nelson reports grants, personal fees and non-financial support from MSD, ViiV Healthcare, Abbvie, BristolMyers Squibb and Gilead Sciences. Alan Winston reports honoraria or research grants from ViiV Healthcare, Gilead Sciences, BMS, Merck and Co. and Janssen. Jaime H Vera reports honoraria and research grants in trials sponsored by Merck, Janssen Cilag, Piramal and Gilead Sciences.

Open Access This article is licensed under a Creative Commons Attribution 4.0 International License, which permits use, sharing, adaptation, distribution and reproduction in any medium or format, as long as you give appropriate credit to the original author(s) and the source, provide a link to the Creative Commons licence, and indicate if changes were made. The images or other third party material in this article are included in the article's Creative Commons licence, unless indicated otherwise in a credit line to the material. If material is not included in the article's Creative Commons licence and your intended use is not permitted by statutory regulation or exceeds the permitted use, you will need to obtain permission directly from the copyright holder. To view a copy of this licence, visit http://creativecommons.org/licenses/by/4.0/.

\section{References}

Ances BM, Clifford DB (2008) HIV-associated neurocognitive disorders and the impact of combination antiretroviral therapies. Curr Neurol Neurosci Rep 8:455-461. https://doi.org/10.1007/s11910-008-0073-3
Apostolova N, Funes HA, Blas-Garcia A, Galindo MJ, Alvarez A, Esplugues JV (2015) Efavirenz and the CNS: what we already know and questions that need to be answered. J Antimicrob Chemother 70:2693-2708. https://doi.org/10.1093/jac/dkv183

Aron AR, Poldrack RA (2006) Cortical and subcortical contributions to stop signal response inhibition: role of the subthalamic nucleus. J Neurosci 26:2424-2433. https://doi.org/10.1523/JNEUROSCI. 4682-05.2006

Beck AT, Ward CH, Mendelson M, Mock J, Erbaugh J (1961) An inventory for measuring depression. Arch Gen Psychiatry 4:561-571. https://doi.org/10.1001/archpsyc.1961.01710120031004

Becker KM, Heinrichs-Graham E, Fox HS, Robertson KR, Sandkovsky U, O’Neill J, Swindells S, Wilson TW (2013) Decreased MEG beta oscillations in HIV-infected older adults during the resting state. J Neuro-Oncol 19:586-594. https://doi.org/10.1007/s13365-0130220-8

Beckmann CF, Smith SM (2004) Probabilistic independent component analysis for functional magnetic resonance imaging. IEEE Trans Med Imaging 23:137-152. https://doi.org/10.1109/TMI.2003. 822821

Buysse DJ, Reynolds CF, Monk TH, Berman SR, Kupfer DJ (1989) The Pittsburgh sleep quality index: a new instrument for psychiatric practice and research. Psychiatry Res 28:193-213. https://doi.org/ 10.1016/0165-1781(89)90047-4

Chang L, Speck O, Miller EN, Braun J, Jovicich J, Koch C, Itti L, Ernst T (2001) Neural correlates of attention and working memory deficits in HIV patients. Neurology 57:1001-1007. https://doi.org/10.1212/ wnl.57.6.1001

Chang L, Yakupov R, Nakama H, Stokes B, Ernst T (2008) Antiretroviral treatment is associated with increased attentional load-dependent brain activation in HIV patients. J NeuroImmune Pharmacol 3:95104. https://doi.org/10.1007/s11481-007-9092-0

Cysique LA, Maruff P, Darby D, Brew BJ (2006) The assessment of cognitive function in advanced HIV-1 infection and AIDS dementia complex using a new computerised cognitive test battery. Arch Clin Neuropsychol 21:185-194. https://doi.org/10.1016/j.acn.2005.07. 011

Dipasquale O, Cercignani M (2016) Network functional connectivity and whole-brain functional connectomics to investigate cognitive decline in neurodegenerative conditions. Funct Neurol 31:191-203. https://doi.org/10.11138/FNeur/2016.31.4.191

Du Plessis S, Vink M, Joska JA, Koutsilieri E, Bagadia A, Stein DJ et al (2015) HIV infection is associated with impaired striatal function during inhibition with normal cortical functioning on functional MRI. J Int Neuropsychol Soc 21:722-731. https://doi.org/10.1017/ S1355617715000971

Elzi L, Erb S, Furrer H, Cavassini M, Calmy A, Vernazza P, Günthard H, Bernasconi E, Battegay M, Swiss HIV Cohort Study Group (2017) Adverse events of raltegravir and dolutegravir. AIDS 31:18531858. https://doi.org/10.1097/QAD.0000000000001590

Ernst T, Chang L, Jovicich J, Ames N, Arnold S (2002) Abnormal brain activation on functional MRI in cognitively asymptomatic HIV patients. Neurology 59:1343-1349. https://doi.org/10.1212/01.WNL. 0000031811.45569.B0

Ernst T, Yakupov R, Nakama H, Crocket G, Cole M, Watters M, Ricardo-Dukelow ML, Chang L (2009) Declined neural efficiency in cognitively stable human immunodeficiency virus patients. Ann Neurol 65:316-325. https://doi.org/10.1002/ana.21594

Filippini N, MacIntosh BJ, Hough MG, Goodwin GM, Frisoni GB, Smith SM et al (2009) Distinct patterns of brain activity in young carriers of the APOE-epsilon4 allele. Pnas 106:7209-7214. https://doi.org/ 10.1073/pnas.0811879106

Fink GR, Marshall JC, Shah NJ, Weiss PH, Halligan PW, GrosseRuyken M, Ziemons K, Zilles K, Freund HJ (2000) Line bisection judgments implicate right parietal cortex and cerebellum as assessed 
by fMRI. Neurology 54:1324-1331. https://doi.org/10.1212/WNL. 54.6.1324

Fink GR, Marshall JC, Weiss PH, Zilles K (2001) The neural basis of vertical and horizontal line bisection judgments : an fMRI study of normal volunteers. Neuroimage 67:59-67. https://doi.org/10.1006/ nimg.2001.0819

Garvey L, Winston A, Walsh J, Post F, Porter K, Gazzard B, Fisher M, Leen C, Pillay D, Hill T, Johnson M, Gilson R, Anderson J, Easterbrook P, Bansi L, Orkin C, Ainsworth J, Palfreeman A, Gompels M, Phillips AN, Sabin CA, For the UK Collaborative HIV Cohort (CHIC) Study (2011) Antiretroviral therapy CNS penetration and HIV-1-associated CNS disease. Neurology 76:693700. https://doi.org/10.1212/WNL.0b013e31820d8b0b

Hoffmann C, Welz T, Sabranski M, Kolb M, Wolf E, Stellbrink HJ, Wyen C (2017) Higher rates of neuropsychiatric adverse events leading to dolutegravir discontinuation in women and older patients. HIV Med 18:56-63. https://doi.org/10.1111/hiv.12468

Jenkinson M, Bannister P, Brady M, Smith S (2002a) Improved optimization for the robust and accurate linear registration and motion correction of brain images. Neuroimage 17:825-841. https://doi. org/10.1016/S1053-8119(02)91132-8

Jenkinson M, Pechaud M, Smith S (2002b) BET2 - MR-based estimation of brain, skull and scalp surfaces. Elev Annu Meet Organ Hum Brain Mapp 17:2002 citeulike-article-id:1179617

Jenkinson M, Beckmann CF, Behrens TEJ, Woolrich MW, Smith SM (2012) FSL - review. Neuroimage 62:782-790. https://doi.org/10. 1016/j.neuroimage.2011.09.015

Knights MJ, Chatziagorakis A, Kumar BS (2017) HIV infection and its psychiatric manifestations: a clinical overview. BJ Psych Adv 23: 265-277. https://doi.org/10.1192/apt.bp.116.016311

Knoch D, Gianotti LRR, Pascual-Leone A, Treyer V, Regard M, Hohmann M, Brugger P (2006) Disruption of right prefrontal cortex by low-frequency repetitive transcranial magnetic stimulation induces risk-taking behavior. J Neurosci 26:6469-6472. https://doi. org/10.1523/JNEUROSCI.0804-06.2006

Kroenke K, Spitzer RL, Williams JBW (2001) The PHQ-9: validity of a brief depression severity measure. J Gen Intern Med 16:606-613. https://doi.org/10.1046/j.1525-1497.2001.016009606.x

Laird AR, Fox PM, Eickhoff SB, Turner JA, Ray KL, Mckay DR et al (2011) Behavioral interpretations of intrinsic connectivity networks. J Cogn Neurosci 23:4022-4037. https://doi.org/10.1162/jocn_a_ 00077

Logan GD, Cowan WB (1984) On the ability to inhibit thought and action: a theory of an act of control. Psychol Rev 91:295-327. https://doi.org/10.1037/0033-295X.91.3.295

McCutchan JA, Wu JW, Robertson K, Koletar SL, Ellis RJ, Cohn S et al (2007) HIV suppression by HAART preserves cognitive function in advanced, immune-reconstituted AIDS patients. AIDS 21:1109 1117. https://doi.org/10.1097/QAD.0b013e3280ef6acd

Mora-Peris B, Laura E, Vera J, Petersen C, Khan M, Dilly Penchala S, et al. (2019) Cerebral function parameters in people living with hiv switching integrase inhibitor. Progr. Conf. Information, CROI 2019, p. Abstract 443

Mumford JA (2012) A power calculation guide for FMRI studies. Soc Cogn Affect Neurosci 7:738-742. https://doi.org/10.1093/scan/ nss059

Pruim RHR, Mennes M, van Rooij D, Llera A, Buitelaar JK, Beckmann CF (2015) ICA-AROMA: a robust ICA-based strategy for removing motion artifacts from fMRI data. Neuroimage 112:267-277. https:// doi.org/10.1016/j.neuroimage.2015.02.064

Rae CL, Hughes LE, Weaver C, Anderson MC, Rowe JB (2014) Selection and stopping in voluntary action: a meta-analysis and combined fMRI study. Neuroimage 86:381-391. https://doi.org/ 10.1016/j.neuroimage.2013.10.012

Sacktor N, Nakasujja N, Skolasky R, Robertson K, Wong M, Musisi S, Ronald A, Katabira E (2006) Antiretroviral therapy improves cognitive impairment in HIV+ individuals in sub-Saharan Africa. Neurology 67:311-314. https://doi.org/10.1212/01.wnl. 0000225183.74521 .72

Schweinsburg BC, Scott JC, Schweinsburg AD, Jacobus J, Theilmann RJ, Frank LR et al (2012) Altered prefronto-striato-parietal network response to mental rotation in HIV. J Neuro-Oncol 18:74-79. https://doi.org/10.1007/s13365-011-0072-Z

Smith SM, Fox PT, Miller KL, Glahn DC, Fox PM, Mackay CE, Filippini N, Watkins KE, Toro R, Laird AR, Beckmann CF (2009) Correspondence of the brain's functional architecture during activation and rest. Proc Natl Acad Sci 106:13040-13045. https://doi.org/ 10.1073/pnas.0905267106

Thomas Yeo BT, Krienen FM, Sepulcre J, Sabuncu MR, Lashkari D, Hollinshead M, Roffman JL, Smoller JW, Zöllei L, Polimeni JR, Fischl B, Liu H, Buckner RL (2011) The organization of the human cerebral cortex estimated by intrinsic functional connectivity. J Neurophysiol 106:1125-1165. https://doi.org/10.1152/jn.00338. 2011

Underwood J, Robertson KR, Winston A (2014) Could antiretroviral neurotoxicity play a role in the pathogenesis of cognitive impairment in treated HIV disease? AIDS 29:253-261. https://doi.org/10. 1097/QAD.0000000000000538

Vallar G, Bello L, Bricolo E, Castellano A, Casarotti A, Falini A, Riva M, Fava E, Papagno C (2014) Cerebral correlates of visuospatial neglect: a direct cerebral stimulation study. Hum Brain Mapp 35: 1334-1350. https://doi.org/10.1002/hbm.22257

Vera JH, Bracchi M, Alagaratnam J, Lwanga J, Fox J, Winston A, Boffito M, Nelson M (2019) Improved central nervous system symptoms in people with HIV without objective neuropsychiatric complaints switching from efavirenz to rilpivirine containing cART. Brain Sci 9. https://doi.org/10.3390/brainsci9080195

Vossel S, Geng JJ, Fink GR (2014) Dorsal and ventral attention systems. Neurosci 20:150-159. https://doi.org/10.1177/1073858413494269

Walker KA, Brown GG (2018) HIV-associated executive dysfunction in the era of modern antiretroviral therapy: a systematic review and meta-analysis. J Clin Exp Neuropsychol 40:357-376. https://doi. org/10.1080/13803395.2017.1349879

Winkler AM, Ridgway GR, Webster MA, Smith SM, Nichols TE (2014) Permutation inference for the general linear model. Neuroimage 92: 381-397. https://doi.org/10.1016/j.neuroimage.2014.01.060

Wise RG, Tracey I (2006) The role of fMRI in drug discovery. J Magn Reson Imaging 23:862-876. https://doi.org/10.1002/jmri.20584

Wu Y, Wang J, Zhang Y, Zheng D, Zhang J, Rong M, Wu H, Wang Y, Zhou K, Jiang T (2016) The neuroanatomical basis for posterior superior parietal lobule control lateralization of visuospatial attention. Front Neuroanat 10. https://doi.org/10.3389/fnana.2016.00032

Zhuang Y, Qiu X, Wang L, Ma Q, Mapstone M, Luque A, Weber M, Tivarus M, Miller E, Arduino RC, Zhong J, Schifitto G (2017) Combination antiretroviral therapy improves cognitive performance and functional connectivity in treatment-naïve HIV-infected individuals. J Neuro-Oncol 23:704-712. https://doi.org/10.1007/s13365017-0553-9

Zigmond AS, Snaith RP (1983) The hospital anxiety and depression scale. Acta Psychiatr Scand 67:361-370. https://doi.org/10.1111/j. 1600-0447.1983.tb09716.x

Publisher's note Springer Nature remains neutral with regard to jurisdictional claims in published maps and institutional affiliations. 